\title{
Das Schaffen günStiger Rahmenbedingungen für KMU Eine vergleichende Untersuchung der Gestaltungsräume ländlicher Gemeinden in Mitteleuropa ${ }^{1)}$
}

\author{
Stephan LoIDL, Richard LANG und Matthias FINK, alle Wien*
}

mit 1 Abb. im Text

\section{INHALT}

Summary

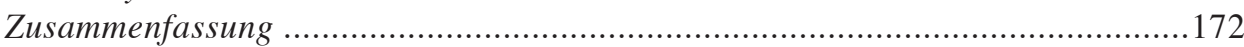

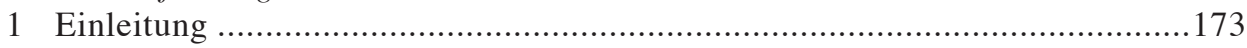

2 Inhaltsanalyse narrativer Interviews als Schlüssel zur Erfassung

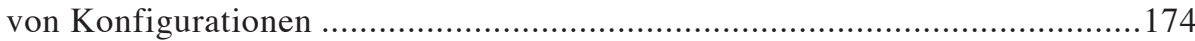

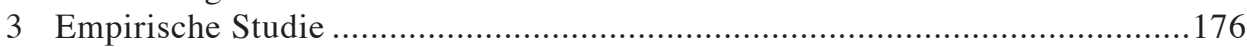

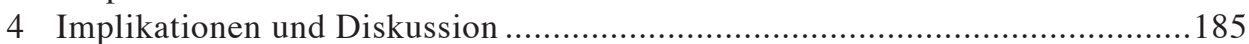

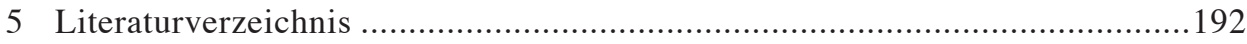

\footnotetext{
1) Dieser Beitrag enthält Auszüge aus Mugler J., Fink M., Loidl St. (2006), Erhaltung und Schaffung von Arbeitsplätzen im ländlichen Raum - Gestaltung günstiger Rahmenbedingungen für Klein- und Mittelbetriebe. Wien, Manz, und Mugler J., Loidl St., Fink M., Lang R., Teodorowicz S. (2008), Zentraleuropäische Gemeindeentwicklung - Gestaltung günstiger Rahmenbedingungen für Klein- und Mittelbetriebe durch grenzüberschreitenden Knowhowtransfer. Wien, Manz.

* Mag. Stephan LoIdL, Institut für Betriebswirtschaftslehre der Klein- und Mittelbetriebe, Wirtschaftsuniversität Wien, Augasse 2-6, A-1090 Wien; e-mail: stephan.loidl@wu.ac.at, http://www.wu.ac.at/kmb; Mag. Richard LANG, Forschungsinstitut für Kooperationen und Genossenschaften (RiCC), Wirtschaftsuniversität Wien, Augasse 2-6, A-1090 Wien; e-mail: richard.lang@wu.ac.at, http://www.wu.ac.at/ricc; Univ.-Ass. MMag. Dr. Matthias FInK, Institut für Betriebswirtschaftslehre der Klein- und Mittelbetriebe Wirtschaftsuniversität Wien, Augasse 2-6, A-1090 Wien; e-mail: matthias.fink@wu.ac.at, http://www. wu.ac.at $/ \mathrm{kmb}$
} 
Summary

Creating favourable conditions for SMEs - A comparative study of the rural municipalities' scope of action in Central Europe

How can municipalities in Central Europe create favourable conditions for local business? What and how can municipalities learn from each other? How can each individual in the local area contribute? What requirements have to be met before know-how can successfully be transferred on a communal level? To answer all these questions, we discuss the manifold opportunities, restrictions and prerequisites of establishing favourable conditions for small and medium enterprises in rural municipalities in Central Europe. First, we derive suggestions for successful intervention measures for municipality development from data collected in 65 intensive research interviews with parties involved (mayors, opposition councillors, entrepreneurs and representatives of citizens' initiatives) from 18 municipalities across Austria. In the next step, it is established which measures - under which conditions - are suitable for know-how transfer to transition countries in East Central Europe bordering Austria (Czech Republic, Slovakia, Hungary and Slovenia) and which barriers have to be overcome. For this purpose, 2,000 questionnaires were disseminated and 61 qualitative interviews conducted in these four countries. The analysis results in 17 theses. We draw implications and discuss the findings against a broader background.

\section{Zusammenfassung}

Wie können Gemeinden günstige Rahmenbedingungen für die lokale Wirtschaftsstruktur schaffen? Wie und was können Gemeinden dabei voneinander lernen? Wie können die einzelnen Bürger in der Kleinregion dazu beitragen? Welche Bedingungen sind für einen erfolgreichen Know-how-Transfer zwischen den Gemeinden Mitteleuropas noch zu schaffen? Um diese Fragen zu beantworten, diskutieren wir zahlreiche Möglichkeiten, Einschränkungen und Voraussetzungen für die Schaffung günstiger Rahmenbedingungen für Klein- und Mittelbetriebe in den ländlichen Gemeinden Mitteleuropas. Zuerst leiten wir aus den im Rahmen von 65 narrativen Interviews mit Betroffenen (Bürgermeistern, oppositionellen Gemeinderäten, Unternehmern und Bürgerinitiativen) aus 18 über Österreich verteilten Gemeinden gewonnenen Daten vielfältige Ansatzpunkte für Interventionen in Gemeindeentwicklungen ab. Daran anschließend untersuchen wir, welche in österreichischen Gemeinden erfolgreichen Maßnahmen unter welchen Voraussetzungen für einen Know-how-Transfer zu Gemeinden in den Nachbarländern Österreichs (Tschechien, der Slowakei, Ungarn und Slowenien) in Frage kommen und welche Barrieren es dabei zu überwinden gilt. Dazu wurden in diesen vier Ländern 2.000 Fragebögen versandt und 61 qualitative Leitfadengespräche geführt. Die Analyse mündet in 17 Thesen. Abschließend formulieren wir Implikationen für die Gemeindeentwicklung und diskutieren die Ergebnisse in einem breiteren Kontext. 


\section{Einleitung}

Gemeinden gestalten in einem erheblichen Ausmaß die Rahmenbedingungen für die Entwicklung von klein- und mittelbetrieblichen Unternehmen (KMU) mit (Europäısche Kommission 2003). Um Arbeitsplätze zu erhalten und zu schaffen, sind Unternehmensstrukturen geringerer Größe gerade im ländlichen Raum unentbehrlich. Diese Unternehmen werden als wirtschaftlicher Motor des ländlichen Raumes gesehen (Mugler 2005). Besonders bei kleinen und mittelgroßen Handwerksunternehmen wurde regionalwirtschaftliche Bedeutung festgestellt (RudolPH 1997). Ein starker und prosperierender KMU-Sektor stellt somit eine wichtige Voraussetzung für die erfolgreiche wirtschaftliche Entwicklung von Regionen dar. Dies gilt verstärkt vor dem Hintergrund der spezifischen Eigenschaften ländlicher Arbeitsmärkte (FASSMANN \& Meusburger 1997). Damit rücken klein- und mittelunternehmerische Strukturen in den Fokus der Regionalentwicklung (BENDER 1997). In der aktuellen betriebswirtschaftlichen Forschung gewinnt der Zusammenhang zwischen Entrepreneurship oder KMU einerseits und der Regionalentwicklung andererseits an Beachtung, was auch durch den Bedeutungsgewinn einschlägiger Publikationsorgane wie Entrepreneurship \& Regional Development dokumentiert wird.

Schon eine oberflächliche Betrachtung zeigt, dass sich beispielsweise die Gemeinden in Österreich höchst unterschiedlich entwickeln. Während in der wirtschaftlich dynamischsten ländlichen Gemeinde Österreichs zwischen den Volkszählungen 1991 und 2001 saldiert rund 1.000 neue Arbeitsplätze geschaffen wurden und sich rund 60 Unternehmen angesiedelt haben, verloren andere Gemeinden fast alle Arbeitsplätze und knapp die Hälfte der ansässigen Unternehmen.

In diesem Beitrag wird den Ursachen für die unterschiedlichen Entwicklungsverläufe in ländlichen Gemeinden Mitteleuropas nachgegangen, um Gestaltungsspielräume als Ansatzpunkte für Maßnahmen zur Schaffung günstiger Rahmenbedingungen für Klein- und Mittelunternehmen zu identifizieren.

Die untersuchten Länder umfassen neben Österreich auch dessen ostmitteleuropäische Nachbarländer Tschechien, Slowakei, Ungarn und Slowenien. Untersuchungseinheiten sind Kleinregionen im ländlichen Raum. Daher wird der Begriff „ländlicher Raum" nicht als homogene Kategorie angesehen, sondern als Oberbegriff für oft auch heterogene Teilräume (SchÖN 1997). Die große Heterogenität der üblicherweise als ländlich bezeichneten Gebiete (ESSMANN 1980) macht seine Charakterisierung in Form einer taxativen Aufzählung von Merkmalen unmöglich. Weder auf Eigenschaften der Räume ausgerichtete sogenannte ,innere“ noch an Messgrößen orientierte „äußere“ Definitionsansätze (Henkel 1993; Du Plessis et al. 2002) konnten bisher einen Konsens schaffen. Die Tatsache, dass ländlichen Räumen zugeschriebene Funktionen nicht in gleichem Maße auf alle ländlichen Gebiete zutreffen oder sie nicht exklusiv dem ländlichen Raum zugerechnet werden können, schränkt auch die Definitionskraft funktionaler Typologien ein (Langosch 1994; Schmalhaus \& Stember 1993). Im Rahmen unserer Untersuchung wurden jene Räume als ländlich klassifiziert, die nach der Definition der Organisation für Wirtschaftliche Zusammenarbeit und Entwicklung (Organisation for Economic Co-operation and Development, OECD 1993; 1995) als ländlich einzustufen sind. 
Wenn wir als räumliche Bezugseinheit der Untersuchung die Kleinregion verwenden, verstehen wir darunter eine Gemeinde samt ihren Verflechtungen zu Nachbargemeinden. Damit sind die jeweiligen Kleinregionen aus der Sicht unterschiedlicher Gemeinden nicht zwangsläufig kongruent. Dies bedeutet aber für unsere Untersuchung keinen Nachteil, auch deshalb nicht, weil sich die von uns untersuchten Kleinregionen ${ }^{2)}$ ohnehin nicht überlappen. Kleinregionen sind nach unserem Verständnis ihrerseits Teile einer Region.

Nachdem wir im zweiten Abschnitt dieses Beitrags die methodische Basis für die Untersuchung geschaffen haben werden, beschreibt der dritte Abschnitt die Datenerhebung und -auswertung und präsentiert die Ergebnisse der empirischen Studie. Vor dem Hintergrund der Diskussion der Ergebnisse werden im vierten Abschnitt dann Implikationen für Forschung und Praxis abgeleitet.

\section{Inhaltsanalyse narrativer Interviews als Schlüssel zur Erfassung von Konfigurationen}

Lange Zeit hat sich die betriebswirtschaftliche Forschung auf die Analyse monokausaler und häufig auch unidirektionaler Beziehungen zwischen Variablen konzentriert. Mit großem Elan und auch Mitteleinsatz führte man umfangreiche Untersuchungen beispielsweise zu gründungs- oder erfolgsrelevanten Faktoren durch (GRICHNIK 2006). Auf der Basis von großen Stichproben unterschiedlicher Qualität entstand so eine Vielzahl von Forschungsergebnissen mit stark eingeschränkter praktischer Relevanz (TsANG \& KwAN 1999). Eine solche Forschung konnte empirisch feststellbare Phänomene kaum fassen und schon gar nicht erklären (Bouckenooghe et al. 2007; Diekmann 2000).

Dass eine vollständige Modellierung des betriebswirtschaftlich relevanten Ausschnitts der Welt unmöglich und auch nicht sinnvoll ist, steht außer Frage. Dennoch ist der Nutzen eines reduktionistischen Forschungsansatzes ebenso fraglich, denn die auf der Basis simplizistischer Modelle generierten empirischen Aussagen können mit der Erlebniswelt der Praktiker nicht ausreichend in Beziehung gesetzt werden (CHANDLER \& LyON 2001; HitT et al. 2004). Sie stellen kaum praxisrelevante Aussagen oder gar Handlungsempfehlungen dar (Gopinath \& Hoffman 1995) und werden daher auch kaum rezipiert (VAN DE VEN 2002).

Konzeptionell kann eine ganzheitliche Perspektive einen möglichen Ausweg aus dieser unbefriedigenden Situation darstellen. Dabei wird versucht, durch eine möglichst umfassende Zusammenstellung von Variablen empirische Phänomene in ihrer Gesamtheit abzubilden. Misst man die Ausprägungen dieser Variablen, so entsteht zum Zeitpunkt der Messung eine Momentaufnahme der Gestalt (Konfiguration) des in den Variablen abgebildeten Wirklichkeitsausschnitts (VeliYath \& SRINIVASAn 1995). In der Differenzanalyse der Konfigurationen zu verschiedenen Zeitpunkten lassen sich

\footnotetext{
2) Aus Gründen des bei der Untersuchung zugesicherten Datenschutzes muss auf die Nennung der untersuchten Kleinregionen verzichtet werden.
} 
dann Entwicklungen identifizieren. Die Veränderung von Variablenbündeln kann so mit Entwicklungen in Verbindung gebracht werden. In diesem Sinn wirken nicht einzelne Variablen auf andere Variablen, sondern es wird in Wechselwirkungen zwischen Variablenbündeln gedacht (WIKLUND \& SHEPHERD 2005). Das Ziel ist die zum Verstehen führende Rekonstruktion von Gestaltveränderungen und eine darauf basierende Ableitung von Ansatzpunkten und Strategien für Interventionen (HARMs et al. 2009).

Ein Fokus auf Variablen, die vor dem Hintergrund der jeweiligen Forschungsfrage relevant sind, ist ein grundlegendes Prinzip der modernen Forschungspraxis. Nur durch diese komplexitätsreduzierende Vorgehensweise können überhaupt aus der unendlichen Zahl an Variablen die interessierenden Zusammenhänge und Entwicklungen identifiziert werden. Es stellt sich dabei jedoch die Frage, welche aus der unendlichen Zahl an möglichen Variablen eben diese relevanten sind. An dieser Frage scheiterte bisher auch der breite Einsatz der Konfigurationstheorie als konzeptioneller Rahmen für empirische Erhebungen (HARMS et al. 2009). Zwar wurde versucht, durch eine theoriegeleitete Definition von Sphären die vor dem Hintergrund der Forschungsfrage relevanten Ausschnitte der Wirklichkeit zu benennen und so eine systematische Suche nach relevanten Variablen zu erleichtern (MugLer 2005). Aber ein solches Vorgehen ist von großen Unsicherheiten geprägt und die Auswahlentscheidungen sind selten stringent zu argumentieren, wodurch das Kriterium der Nachvollziehbarkeit wissenschaftlicher Arbeit verletzt wird.

Erst die Auswahl der Variablen lässt das Forschungsobjekt entstehen. Durch die Auswahl der zu messenden Aspekte der Wirklichkeit konstruiert der Forscher sein Forschungsobjekt. Damit stellt die Variablenwahl einen entscheidenden Schritt im Forschungsprozess dar (FINK 2005). Wenn nun aber die theoriegeleitete Auswahl nicht stringent argumentierbar ist, dann kann nur auf eine empirische Basis zurückgegriffen werden. Quantitative Methoden der Datenerhebung setzen eine a priori bestehende Sinnstruktur bezüglich des Forschungsobjekts voraus. Daher kann eine solche empirische Basis nur mittels qualitativer Datenerhebung geschaffen werden, da nur offene Methoden nicht vorstrukturierte Daten ermöglichen (Diekmann 2000). Besonders das narrative Interview ermöglicht es im Feld zu erheben, ohne von Seiten des Forschers Strukturen vorzugeben. Die Strukturierungsleistung wird von den Befragten selbst erbracht. Damit kann die Struktur des Forschungsgegenstandes aus den Transkripten erarbeitet werden (SCHÜTZE 1987). Dafür bietet sich die Inhaltsanalyse nach MAYRING (2002) an. Sie ermöglicht die Identifikation der hinter den Daten stehenden Sinnstrukturen. So können die dem Feld inhärenten Strukturen aufgedeckt werden (RUST 1981).

Bezüglich der Auswahlproblematik bietet die Inhaltsanalyse von Narrationen der Akteure im interessierenden Feld die Möglichkeit, deren Strukturierungsleistung zu nutzen und so eine a posteriori-Auswahl der Variablen vorzunehmen. Damit bildet sich erst im Rahmen der Inhaltsanalyse nach und nach ein Variablengerüst (das Kategorienschema) heraus, das dann den Bezugsrahmen der Untersuchung darstellt. Die Betroffenen definieren das Untersuchungsobjekt als in der Konfiguration abgebildete Struktur selbst. Die Variablen bilden daher zwangsläufig den untersuchten Ausschnitt der Wirklichkeit ab, sind also relevant.

Das in dieser Studie implementierte Forschungsdesign macht die direkte Anwendung des Konfigurationsansatzes in empirischen Studien möglich. Die Unsicherheit der 
Auswahlentscheidung wird durch die Nutzung der Offenheit qualitativer Datenerhebungs- und interpretativer Auswertungsmethoden zu einer Stärke des ganzheitlichen Ansatzes, der damit deutlich an Relevanz für die empirische Sozialforschung gewinnt.

\section{Empirische Studie}

\subsection{Datenerhebung}

In Österreich wurden zwischen September 2005 und Februar 2006 in 18 Kleinregionen in allen acht Bundesländern mit ländlichen Räumen (das heißt außer Wien) mit insgesamt 65 Gesprächspartnern 4.157 Minuten Interviews geführt. 1.473 Seiten Transkript entstanden.

Da keine repräsentative Abbildung der Kleinregionenstrukturen in Österreich, sondern eine Erklärung typischer Entwicklungsverläufe angestrebt war, wurden nicht zufallsgesteuert Kleinregionen untersucht. Es wurden vielmehr Kleinregionen ausgewählt, die erstens zwischen den Arbeitsstättenzählungen der Jahre 1991 und 2001 eine ausgeprägte $\mathrm{Zu}$ - oder Abnahme von Arbeitsplätzen und anderer in unserem Zusammenhang wichtiger Merkmale aufwiesen sowie zweitens von einer ausgeprägten Merkmalskategorie gekennzeichnet waren, der Einfluss auf die (positiven oder negativen) Entwicklungsverläufe von Kleinregionen zugeschrieben wird. Dabei wurden unter anderem folgende Merkmalskategorien berücksichtigt: Bekanntheit der Region für ein bestimmtes Produkt; sozialer Zusammenhalt; Umbruch der Wirtschaftsstruktur; interkommunales Betriebsgebiet. Für jede dieser Merkmalskategorien wurden jeweils zwei Kleinregionen identifiziert, von denen eine im Betrachtungszeitraum eine gute, die andere eine schlechtere Entwicklung genommen hatte.

In den ostmitteleuropäischen Nachbarländern erfolgte die Erhebung ein Jahr später. Auch hier wurden die Daten im Rahmen unmittelbarer Feldarbeit erhoben. Die narrativen Interviews wurden in der jeweiligen Landessprache geführt. Insgesamt wurden in den vier Ländern mit 61 Gesprächspartnern Interviews in einer Gesamtlänge von 3.812 Minuten geführt, die in 1.142 Seiten Transkript resultierten.

Es wurden insgesamt zehn ländliche Kleinregionen analysiert. Die Zahl pro Land richtete sich nach der Staatsfläche, wobei jedes Land durch zumindest zwei Kleinregionen vertreten sein sollte. Folglich wurden jeweils drei tschechische und ungarische sowie jeweils zwei slowakische und slowenische Kleinregionen untersucht. Um Einflüsse unterschiedlicher Regelungen auf regionaler Verwaltungsebene zu erfassen, wurde bei der Auswahl der Kleinregionen darauf geachtet, dass die Fälle nach Einheiten auf NUTS-2-Ebene ${ }^{3)}$ in verschiedenen Regionen liegen. Außerdem sollte der Einfluss der

\footnotetext{
3) Die Ebene NUTS-2 der Systematik der Gebietseinheiten für die Statistik (Nomenclature des unités territoriales statistiques) der Europäischen Union besteht in Tschechien aus acht, in Ungarn aus sieben und in der Slowakei aus vier Einheiten. Slowenien ist auf dieser Ebene nicht unterteilt. In keinem der drei erstgenannten Staaten entspricht die NUTS-2-Gliederung Gebietskörperschaften. Es handelt sich also durchwegs um reine Planungs- und Statistikregionen.
} 
Entfernung der untersuchten Kleinregionen von der österreichischen Grenze untersucht werden. Daher wurde bei der Auswahl darauf geachtet, dass in jedem untersuchten Land zumindest eine Kleinregion in der Nähe der österreichischen Grenze liegt und zumindest eine davon weiter entfernt.

Nach diesen Einschränkungen wurden die Kleinregionen mit der Hilfe von Experten aus den jeweiligen Ländern zur Analyse ausgewählt. Um eine möglichst große Reichweite der generierten Aussagen zu gewährleisten, sollten die ausgewählten Kleinregionen besonders hinsichtlich ihrer demographischen und wirtschaftlichen Eckdaten typisch für die jeweilige NUTS-2-Region sein. Auch bezüglich qualitativer Kriterien wie der historischen Entwicklung und der sozialen Situation sollten die ausgewählten Kleinregionen typisch für die jeweilige Region sein.

Innerhalb der Kleinregionen wurde der ganzheitlichen Sichtweise folgend versucht, die Perspektive möglichst unterschiedlicher Akteure zu erfassen. So wurden jeweils zumindest der Bürgermeister, ein Gemeinderat der stärksten oppositionellen Fraktion, ein Unternehmer sowie ein Vertreter der mit Regionalentwicklung befassten Institution auf interkommunaler oder regionaler Ebene befragt.

\subsection{Datenauswertung}

Die Interpretation der auf Tonträger aufgezeichneten Interviews erfolgte vierfach und in zwei Stufen. In einer ersten Stufe wurden die Interviews unabhängig voneinander von den Interviewern selbst, im Rahmen eines Seminars in Gruppenarbeiten durch qualifizierte Studenten der Speziellen Betriebswirtschaftslehre der Klein- und Mittelunternehmen an der Wirtschaftsuniversität Wien sowie durch die Autoren interpretiert. Diese drei Interpretationen wurden sodann in einer zweiten Stufe (Interpretation der Interpretationen) in einem diskursiven Prozess zu einer endgültigen Interpretation zusammengeführt. Im Falle von Interpretationsdiskrepanzen wurde eine FeedbackSchleife eingebaut. Im Zuge der Interpretation bildete sich das in Abbildung 1 in Auszügen dargestellte Kategorienschema heraus.

\subsection{Ergebnisse}

Gestaltungsraum Beziehung zwischen Gemeinde und Unternehmerschaft: mehr Miteinander und Füreinander statt Nebeneinander und Gegeneinander

Für den gesamten Untersuchungsraum wurde festgestellt, dass die Kommunikation zwischen Gemeindevertretung und ansässiger Unternehmerschaft nachhaltig gestört ist. Dies gilt besonders für die ostmitteleuropäischen Nachbarländer. Jene wenigen Unternehmer, die auf Unterstützung seitens der Gemeinde hoffen, fühlen sich von der Gemeinde kaum bis gar nicht unterstützt. Die Gemeinden scheinen sich wiederum für die Entwicklung und Situation der Unternehmen kaum zu interessieren. Bemüht sich die Gemeinde Unternehmen zu fördern, stößt sie jedoch schnell an ihre Grenzen. Zugleich wird die Eigeninitiative der Unternehmer zur Lösung oft strukturell bedingter Probleme eingefordert. 


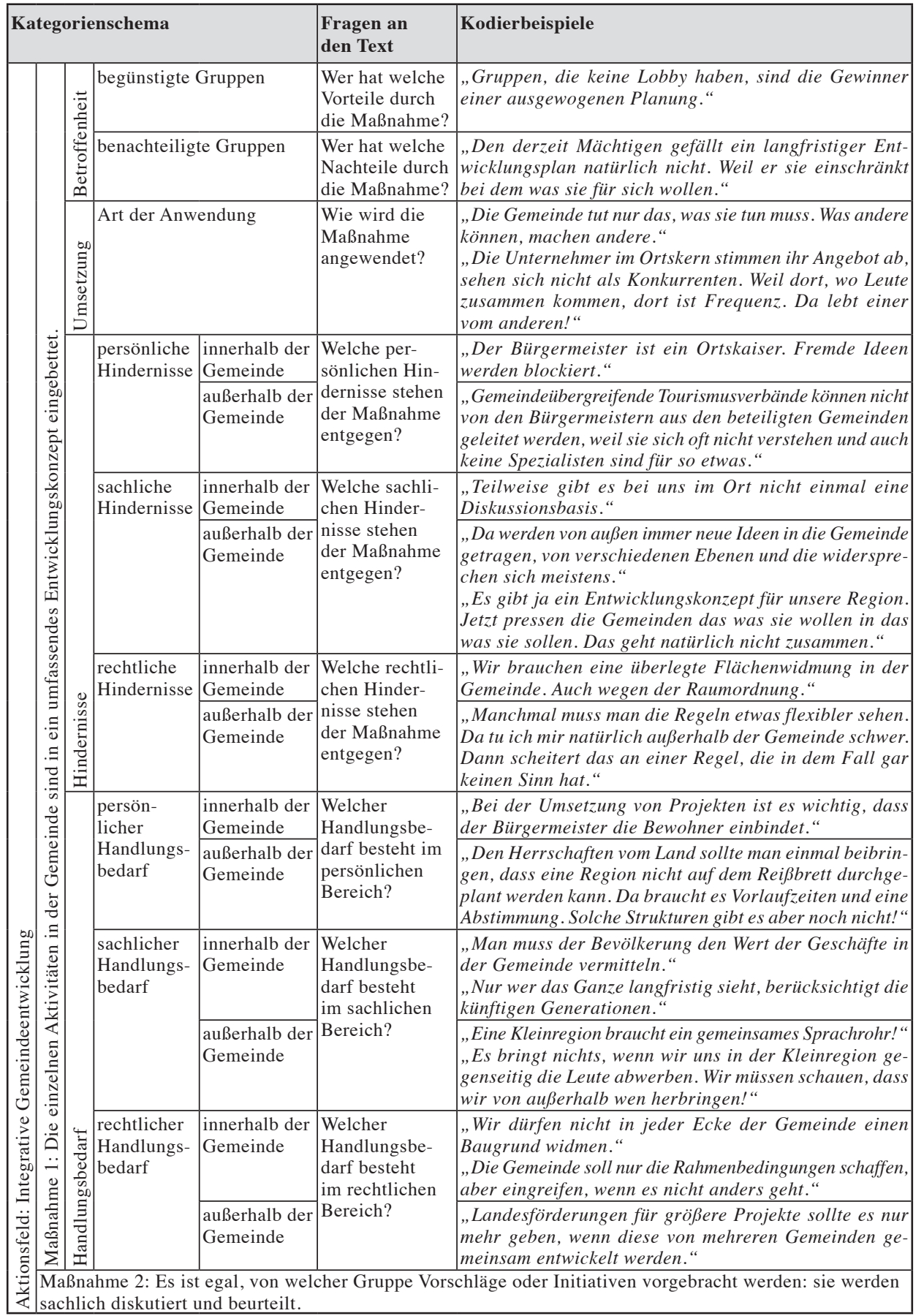

Quelle: Eigene Darstellung

Abb. 1: Auszug aus dem Kategorienschema mit Kodierungsbeispielen 
Eine zentrale Ursache für die problematische Beziehung zwischen Gemeinde und Unternehmern in den ostmitteleuropäischen Ländern liegt im nach der Wende etablierten Glauben an die Selbstregulierungskraft einer extrem liberal ausgeprägten Marktwirtschaft, der bei allen Beteiligten spürbar ist. So erwarten sich die Unternehmer oft gar nicht, dass die Gemeinde und generell die Politik etwas Sinnvolles für sie tun kann. Man mischt sich nicht in die Angelegenheiten der Gemeindeentwicklung ein und verlangt von der Kommunalpolitik dasselbe, wenn es um Fragen der Unternehmensentwicklung geht. Die Gemeinden wiederum sind erst seit zwei Jahrzehnten mit freiem Unternehmertum konfrontiert und können mit ihrer neuen Rolle am freien Markt noch nicht umgehen. Die Bürgermeister mischen sich daher nach Möglichkeit nicht in Angelegenheiten der Unternehmen ein.

Grundsätzlich sehen sich die Unternehmer aber sehr wohl als Teil der sozialen Gemeinschaft „Gemeinde“. Die Wirtschaftsstruktur ist dabei allerdings gedanklich mit den anderen Lebensbereichen in der Gemeinde kaum verwoben. Im Gegenteil, es wird eine bewusste Trennung zwischen unternehmerischer und sozialer Sphäre gelebt. In Ungarn und Slowenien, wo es auch während des Kommunismus bedingt freies Unternehmertum gab, sind leichte Nuancierungen feststellbar: Unternehmen werden hier noch eher als wichtiger Teil des Gemeindelebens wahrgenommen. Dennoch fehlt eine echte Tradition gezielter Förderung klein- und mittelunternehmerischer Strukturen durch die Gemeinde ebenso wie die Wahrnehmung sozialer Verantwortung durch die Unternehmer.

Gestaltungsraum öffentliche Infrastruktur: die Infrastruktur an die Bedürfnisse der Region heranführen

Während die Infrastrukturausstattung von den Betroffenen in Österreich als ausreichend bis gut bewertet wird, sind die teilweise immer noch schwerwiegenden infrastrukturellen Defizite im ländlichen Raum der ostmitteleuropäischen Länder ein Relikt und eine Hypothek der kommunistischen Phase. Ehemalige Prestigeprojekte im Bereich von Verkehrswegen, Gebäuden oder Kommunikationseinrichtungen wurden nicht nach den Bedürfnissen der Bevölkerung entwickelt und entsprechen nicht den Anforderungen an eine moderne technische Infrastruktur. Auch im Bereich der Ver- und Entsorgungsinfrastruktur sind die ländlichen Gemeinden durch Defizite gekennzeichnet. Aufgrund von Budgetrestriktionen können diese nur langsam behoben werden.

Eine moderne Kommunikationsinfrastruktur - im Speziellen das Internet - ist in den ostmitteleuropäischen Ländern vor allem in Privathaushalten noch nicht so verbreitet wie in Österreich oder anderen Ländern des westlichen Europas. Damit sind erhebliche Wettbewerbsnachteile für die Gemeinden, vor allem auch für die dort ansässigen Unternehmen verbunden. Möglicherweise kann jedoch der Schritt des Aufbaus einer festen Infrastruktur gänzlich übersprungen werden, indem flexible Lösungen wie mobiles Internet oder UMTS forciert werden.

Von großer Bedeutung sind staatliche Förderungen für die Renovierung von Gebäuden und das Beheben von Verwahrlosung in den Gemeinden der ostmitteleuropäischen Länder. Für Eigeninitiative in diesem Bereich ist besonders die Identifizierung von Bevölkerung und Unternehmerschaft mit der Gemeinde von Bedeutung. Gleichzeitig wirken eine moderne technische Infrastruktur und ein gepflegtes Ortsbild für die 
Bevölkerung und die Unternehmerschaft identitätsstiftend. Von der Verbesserung der technischen Infrastruktur können so auch indirekte Impulse für die lokale Wirtschaft ausgehen.

Gestaltungsraum sozialer Zusammenhalt: das Gemeinschaftsgefühl aktiv stärken und für Initiativen nützen

Der soziale Zusammenhalt ist in den Gemeinden des Untersuchungsraums stark ausgeprägt. Es existiert eine große Anzahl von Orten der sozialen Begegnung, die gut besucht sind und von den Gemeinden auch substanziell gefördert werden. Pläne für eine nachhaltige soziale Entwicklung auf Gemeindeebene werden jedoch kaum ausgearbeitet.

In Tschechien und in der Slowakei beruht die starke Gemeinschaft unter anderem auch auf der erfolgreichen Vereinstradition im Sport, wobei Fußball und Eishockey an vorderster Stelle zu nennen sind. Schon die Jugend ist durch diese Vereine stark in die Gemeinschaft eingebunden und auch stark heimatverbunden. In diesem Zusammenhang ist speziell die nationale Identität stiftende Tradition der Sportvereine in den slawischen Untersuchungsländern zu erwähnen.

In Slowenien und Ungarn sind es die Kulturhäuser, die ein Zentrum des Gemeindelebens darstellen. Es erscheint wichtig diese Einrichtungen zu erneuern, attraktiver zu gestalten und sie aktiv zur Förderung der Gemeindeentwicklung zu nutzen. So hat beispielsweise das öffentliche Angebot von Internet vielen Gemeindezentren eine neue Bedeutung als sozialer Treffpunkt eingebracht.

Wie auch in Österreich kommt gerade in Slowenien den Gasthäusern für den sozialen Zusammenhalt große Bedeutung zu. Man geht gemeinsam ins Gasthaus und diskutiert dort Fragen der Gemeindeentwicklung. Über die kommunistische Zeit mit ihren eigenen politisch ausgerichteten Vereinsstrukturen hinweg hat sich ein traditionelles Vereinsleben (Heimatkultur) erhalten. Es wird heute teilweise revitalisiert.

Gestaltungsraum regionale Produkte: hochwertige Erzeugnisse mit regionaler Identität anreichern

Um die Wirtschaft in den untersuchten Gemeinden anzukurbeln und nach außen hin ein wettbewerbsfähiges Image zu etablieren, wird die Förderung regionaler landwirtschaftlicher und handwerklicher Produkte, insbesondere von Lebensmitteln vor allem in den ostmitteleuropäischen Ländern als ein kommunalpolitisches „Patentrezept" angesehen. Während aber in Österreich bereits differenzierte Konzepte entwickelt werden, wird diese Strategie in den ostmitteleuropäischen Ländern häufig wenig reflektiert und ohne die notwendigen strukturellen Grundlagen angewendet. So mangelt es dort derzeit noch an Konsumbewusstsein sowie vor allem an Kaufkraft. Damit findet sich noch kein ausreichendes Marktpotenzial für hochwertige regionale Produkte. Das derzeitige Angebot an regionalen Spezialitäten richtet sich damit vor allem an zahlungskräftige (ausländische) Touristen.

In den nächsten Jahren wird sich aber sicher auch in den ostmitteleuropäischen Ländern, so wie es in Österreich in den letzen Jahren zu beobachten war, ein lokaler Markt für regionale Produkte entwickeln. Als Reaktion auf die Allgegenwärtigkeit internationaler Diskontmärkte mit ihrem qualitativ wenig transparenten Angebot scheint 
sich eine Konsumentenbewegung für nachvollziehbare Produkte und schonende Produktionsverfahren zu entwickeln. Dementsprechend könnte die aktuelle oberflächliche Affinität der Konsumenten zu westlichen Produkten in den nächsten Jahren sinken.

Gestaltungsraum Beratung von Unternehmen: Gemeinden und regionale Beratungsinstitutionen entfalten ihre Wirkung im Zusammenspiel

Durch die in den ostmitteleuropäischen Ländern seit der Wende und besonders durch ihre Mitgliedschaft in der Europäischen Union (EU) ausgelöste Dynamik innerhalb der Verwaltungsstrukturen, wie sie an den Beispielen Sloweniens oder Ungarns gut zu beobachten ist, wird die Beratung von Unternehmern erschwert. Die Gemeinde weiß oft nicht, welche Institutionen für welche Unternehmensfragen tatsächlich zuständig sind oder welche Person auf eine konkrete Frage des ansiedlungswilligen Unternehmers tatsächlich kompetent Antwort geben kann. Häufig sind es gleich mehrere Institutionen, die auf unterschiedlichen Verwaltungsebenen für die Ansiedlung von Unternehmern zuständig sind.

Erschwerend kommt in den ostmitteleuropäischen Ländern und teilweise auch in Österreich hinzu, dass Unternehmer und Gemeinde wenig aufeinander zugehen. Nur bei akuten Anlässen wird der Kontakt gesucht. So spricht die Gemeinde die ansässigen Unternehmen an, wenn sie Geld, z.B. für Vereine, benötigt. Wenn Bürgermeister regelmäßige Treffen zur Verbesserung der interkommunalen Kommunikation vorschlagen, reagieren die Unternehmer eher verwundert.

Trotz des geringen finanziellen und teilweise auch rechtlichen Spielraums sind es oft die Bürgermeister selbst, die sich bei der Unternehmerberatung engagieren. Sie begleiten den Unternehmer bei Behördenwegen, sondieren mit ihm ein Baugrundstück und handeln die Ansiedlungskonditionen aus. Bei ihren Beratungsaktivitäten überschreiten sie das eine oder andere Mal ihre tatsächlichen Kompetenzen, wobei diese eben nicht für alle Beteiligten transparent geregelt sind.

Konterkariert werden derartige ambitionierte Einzelinitiativen von Gemeindepolitikern durch den starken Wettbewerb unter den Gemeinden im gesamten Untersuchungsraum. Dies gilt speziell, wenn es um Neuansiedlungen von Betrieben geht. So haben ansiedlungswillige Investoren längst erkannt, dass die Bürgermeister einer Kleinregion leicht gegeneinander auszuspielen sind. Hier ist ein Umdenken sowohl auf kommunaler als auch auf übergeordneter Ebene erforderlich. Die interkommunale Abstimmung solcher Projekte muss als Voraussetzung für die Zuteilung von öffentlichen Unterstützungen definiert werden.

Als Ansprechpartner für Unternehmen und Gemeinden kommt den verschiedenen Organisationen der Regionalberatung eine zentrale Rolle in der Gemeindeentwicklung $\mathrm{zu}$. So sind sie die treibende Kraft hinter der Implementierung endogener Entwicklungsinstrumente (z.B. Bürgerforen), die mehr Pluralismus und basisdemokratische Mitbestimmung in die Gemeinden bringen können. Regionalentwicklungsagenturen fungieren aber genauso als Ansprechstelle für Unternehmer, fördern die Ausarbeitung von nachhaltigen landwirtschaftlichen und touristischen Konzepten und versuchen, die Gemeinden mit Tools der Strategiefindung und -umsetzung in verschiedenen Politikbereichen vertraut zu machen. 
Neben staatlichen Förderprogrammen ist es vor allem das Leader-Programm der Europäischen Union, welches diese wichtigen Beratungsstrukturen für die ländliche Entwicklung in den ostmitteleuropäischen Ländern ermöglicht und wesentliche Beratungsinhalte definiert. Durch ihre europaweite Vernetzung betreiben die Regionalentwickler damit auch einen regen Know-how-Transfer von den alten EU-Mitgliedsländern in die neu beigetretenen Länder. Innerhalb des Interreg-Netzwerks gibt es beispielsweise in den Grenzregionen Österreichs zu den ostmitteleuropäischen Ländern auch eine verstärkte Zusammenarbeit zwischen Regionalberatungen auf beiden Seiten der Staatsgrenze. Auf Gemeindeebene ist ein solcher Know-how-Transfer dagegen noch nicht institutionalisiert, sondern findet allenfalls in Form von informellen Kontakten statt.

Festzuhalten bleibt, dass Regionalberatungen durch ihre Fachkompetenz, ihre tendenzielle politische Unabhängigkeit und ihre jungen Beraterstäbe inzwischen im gesamten Untersuchungsraum zu einem wichtigen Partner für die Gemeinden in vielen Entwicklungsfragen geworden sind. Die Berater selbst sehen ihre Arbeit vor allem durch zu geringe Budgetmittel und bürokratische Hürden behindert.

Gestaltungsraum pluralistische Gemeindeentwicklung: die Bürger zur politischen Mitbestimmung und Eigenverantwortung motivieren

Die Ergebnisse der Studie legen den Schluss nahe, dass eine positive Gemeindeentwicklung im Untersuchungsraum eng mit der Person des Bürgermeisters verknüpft ist. Insbesondere in kleinen Gemeinden trifft dieser viele Entscheidungen autonom und steht beispielsweise auch in direktem Kontakt mit ansiedlungswilligen Unternehmern. Aktivitätsniveau, Demokratieverständnis, unternehmerisches Denken oder Mut zum „Blick über den Tellerrand“ hängen in den Gemeinden der ostmitteleuropäischen Länder im Wesentlichen davon ab, ob ein Kommunalpolitiker bereits vor 1989 politisch aktiv war oder seine politische Laufbahn oder vielleicht sogar seine Ausbildung erst nach der Wende begonnen hat.

Gemeindeentwicklung im Untersuchungsraum als Generationenfrage zu thematisieren erscheint auch insofern als relevant, als die junge Politikergeneration der interkommunalen Zusammenarbeit, der externen Regionalberatung und damit der Anwendung neuer Politikkonzepte in der Regel offener gegenübersteht. Neben Maßnahmen zur Ansiedlung von Unternehmen betrifft dies vor allem die Etablierung pluralistischer und auch transparenter Entscheidungsstrukturen in der Gemeinde. Gerade deshalb erscheint es wichtig, das Interesse der jungen Bevölkerung an der Gemeindeentwicklung insgesamt und speziell an der Kommunalpolitik zu wecken.

Viele Aktivitäten in der Regional- und Gemeindeentwicklung vollziehen sich „top-down“ und werden nicht von der lokalen Bevölkerung (mit)getragen. Dies gilt besonders für die ostmitteleuropäischen Länder. In der kommunistischen Zeit waren endogene Entwicklung, persönlicher Einsatz und regionale Kooperation verpönt, da die Zentralregierungen Angst vor Systemkritikern hatten. Die damals angelernte Passivität wird durch kulturelle Merkmale modifiziert, wodurch sich unterschiedliche Ausprägungen ergeben.

Die Zivilgesellschaft in den ostmitteleuropäischen Ländern ist allerdings vielerorts lebendiger als man aufgrund dieser Prägungen annehmen würde. Es fehlt ihr aber oft 
an dauerhafter Organisation, was eine stärkere Einbindung in regionalpolitische Prozesse erschwert. Gemeinden warten oft auf Vorschläge der Bürger, fördern aber nicht die organisatorischen Grundlagen dafür. Gemeindevertreter aktivieren und nützen zu wenig die ausgeprägte Fähigkeit der Bevölkerung zur Selbstorganisation. Diese hat sich in kommunistischer Zeit in Form von weit verbreiteten informellen Netzwerken ("opaque networks") gezeigt. Sie waren damals notwendig, um die Ineffizienz des politischen Systems auszugleichen. Auch bezogen auf heute liegt der Schluss nahe, dass selbstorganisierte Gruppen mit starken Promotoren in einzelnen Bereichen der Regionalentwicklung (z.B. Dorferneuerung, soziale Aktivitäten) Aufgaben besser und schneller erledigen könnten als die Gemeindeverwaltung.

Die erfolgreiche Implementierung demokratischer und pluralistischer Entscheidungsprozesse ist eng mit der Person des Bürgermeisters und mit der Politikergeneration, der sie entstammt, verknüpft. Dies umso mehr, als es in den Gemeinden des ganzen Untersuchungsraums zumeist jeweils eine deutlich dominierende parteipolitische Gruppierung gibt. Von der politischen und weltanschaulichen Grundeinstellung sowie von der Führungsqualität des Bürgermeisters hängt es ab, ob in der Gemeinde das richtige Gleichgewicht gefunden wird, ob es gelingt, Opposition, Bürgerlisten, Unternehmer und Bevölkerung in Entscheidungsprozesse einzubeziehen, aber auch ob der Bürgermeister in der Lage ist wichtige Entscheidungen autonom zu treffen und sie konsequent gegen externe Einflussnahme durchzusetzen.

Gestaltungsraum Unternehmensgründung und Innovation: betriebliche Innovation und regionale Unternehmenskooperation ermöglichen

Die gezielte Unterstützung von Neugründungen von Klein- und Mittelunternehmen wird seitens der Gemeinden kaum forciert. So werden Gründungsunternehmer vor allem durch Regionalberatungen unterstützt. Daneben existieren Inkubatoren sowie Industrieparks, die sich allerdings zumeist bereits im Umfeld von Ballungsräumen befinden. Was fehlt, sind regionale Strategien der Gründungs- und Innovationsförderung, die auf die Besonderheiten des ländlichen Raums Rücksicht nehmen.

Eine als Patentlösung fehlinterpretierte Strategie, um Innovationen und Arbeitsplätze in Kleinregionen zu schaffen, zeigt sich vielerorts im Versuch, Großbetriebe anzuziehen und diese mit den ansässigen produzierenden Betrieben zu vernetzen. Die Notwendigkeit einer diversifizierten lokalen Wirtschaftslandschaft wird oft nicht gesehen; ebenso nicht die Problematik der durch diese Strategie entstehenden Abhängigkeit von einzelnen Großbetrieben und Branchen. Für Beschäftigte aus sterbenden Industriezweigen und Großbetrieben gibt es kaum wirtschaftliche Auffangnetze, weil die Wirtschaftsstrukturen in vielen ländlichen Räumen bis heute nicht diversifiziert wurden.

Ein wesentliches Hindernis für eine aktive Ansiedlungspolitik der Gemeinden und Regionalberatungen ist die Abwanderung qualifizierter Arbeitskräfte in die Städte und in den ostmitteleuropäischen Ländern vor allem ins Ausland. Zusätzlich mindert die zumeist schwach ausgebaute und veraltete Verkehrsinfrastruktur der Transformationsländer die Attraktivität der Standorte im ländlichen Raum. In Slowenien behindern überdies die - besonders in touristischen Gebieten - hohen Grundstückspreise die Neuansiedlung von Unternehmen. 
Als besonders problematisch erscheint, dass sich die Gemeinden in ihrer Wirtschaftspolitik nicht abstimmen. Es fehlt sogar oft jegliches Verständnis der Entscheidungsträger für die Notwendigkeit interkommunaler Zusammenarbeit. Wenn es funktionierende interkommunale Betriebsgebiete gibt, dann in der Nähe der Ballungszentren. Derartige Projekte werden jedoch nicht auf lokaler Ebene entwickelt, und man strebt keine gemeindeübergreifende Lösung an. So handelt es sich im ländlichen Raum bei Gewerbezonen oft um Insellösungen, die wegen ihrer fehlenden Einbettung in die sie umgebenden Strukturen von den Unternehmen dann auch kaum angenommen werden. Die extreme Wettbewerbshaltung der Gemeinden in Fragen der Standortpolitik verleiht den Investoren zudem große Verhandlungsmacht. Die Möglichkeit, die Gemeinden in Verhandlungen gegeneinander auszuspielen, schadet der Kleinregion.

Unsere Studie zeigt, dass die Landwirtschaft allein schon wegen der grundlegenden strukturellen Gegebenheiten auch in Zukunft eine entscheidende Rolle für die Gemeindeentwicklung spielen wird. Hier gilt es kooperative Strategien zu finden, um eine wettbewerbsfähige kleinbetrieblich strukturierte Landwirtschaft zu erhalten. Als zielführend erscheinen in diesem Zusammenhang beispielsweise branchenübergreifende Kooperationen, die sich auf die Produktion regionaler Besonderheiten in den Bereichen Lebensmittel und Handwerk konzentrieren, wodurch die beteiligten Unternehmen auch eine verbesserte Wettbewerbsposition gegenüber ausländischen Handelsketten einnehmen könnten.

Gestaltungsraum interkommunale Kooperation: mehr Zusammenhalt und weniger Konkurrenzdenken zwischen Gemeinden stärken Kleinraum und Region

Die Notwendigkeit der interkommunalen Zusammenarbeit in der Gemeindeentwicklung wird von den Entscheidungsträgern öffentlich bekundet. Konsequent umgesetzt wird der Gedanke allerdings nur im Bereich des Tourismus. Hier leuchten den Verantwortlichen die Vorteile der Bündelung von Ressourcen ein. Fortschritte und Erfolge sind relativ schnell erkennbar. Ansatzweise findet interkommunale Kooperation auch in den Bereichen Infrastruktur, Sozial- und Bildungswesen sowie Kultur statt. Hinter jedem positiven Kooperationsbeispiel stehen allerdings immer auch monetäre Anreize durch Förderprogramme und starke Promotoren. Des Weiteren geht es bei der interkommunalen Kooperation unterschwellig auch um Prestigegewinn für die Gemeinde(n) und die Bürgermeister.

In den ländlichen Gemeinden der ostmitteleuropäischen Länder wird der Wettbewerbsgedanke häufig übertrieben. Während in Österreich so wie in vielen anderen traditionellen Marktwirtschaften Europas in der Regionalpolitik kooperative Elemente wieder stärker Beachtung finden, sieht man in den ostmitteleuropäischen Ländern den Wettbewerb in fast allen Politikfeldern als rein positiv besetzte Handlungsmaxime. Das übertriebene Wettbewerbsdenken ist auch die Folge einer jahrzehntelangen willkürlichen Verteilungspolitik der kommunistischen Regime. 


\section{Implikationen und Diskussion}

\subsection{Implikationen}

Im Gestaltungsraum „Beziehung zwischen Gemeinde und Unternehmerschaft“ sollten die Gemeinden im Untersuchungsraum versuchen, der Unternehmerschaft mehr Wirtschaftskompetenz zu signalisieren, um zukünftig als zuverlässiger Partner in Fragen der Wirtschafts- und damit auch Unternehmensentwicklung wahrgenommen zu werden. Dies gelänge beispielsweise durch die Schulung oder Anstellung von kompetenten Ansprechpersonen für Unternehmer, welche bei Spezialfragen auch an die richtigen Stellen (z.B. regionale Wirtschaftskammer oder Regionalentwicklungsagenturen) weiter verweisen können. Unterschwellig werden in den untersuchten Transformationsländern viele Gemeindevertreter mit dem (alten) System assoziiert, welches als Antithese zur Marktwirtschaft und damit als unternehmerfeindlich gilt. Um hier ein neues „Weltbild“ bei den Beteiligten zu etablieren, gilt es regelmäßige Treffen zwischen Gemeindevertretung und lokalen Unternehmern zu organisieren, die von Externen moderiert werden. Ohne eine tragfähige Beziehung zwischen Gemeinde und Unternehmerschaft werden die Entwicklungsprobleme im ländlichen Raum unter den derzeitigen makropolitischen Rahmenbedingungen nur schwer zu lösen sein.

Im Gestaltungsraum „öffentliche Infrastruktur“ kann durch flächendeckende Verbreitung moderner Kommunikations- und Informationstechnologie ein wichtiger Erfolgsfaktor für betriebliche Innovationen im ländlichen Raum geschaffen werden. Zudem stellt das Internet eine dezentrale Kommunikationsplattform für die Regionalentwicklung dar. Es ist „ein Fenster zur Welt“ für die lokale Bevölkerung. Der Ausbau von Verkehrswegen würde im ländlichen Raum angesiedelten Unternehmen auch eine bessere reale Verbindung zur Außenwelt gewährleisten und damit Standortnachteile entschärfen.

Darüber hinaus ist eine moderne und bedarfsgerechte soziale Infrastruktur ein wesentlicher Faktor, um die Gemeinde als attraktiven Lebensraum zu positionieren und so die junge Bevölkerung im Ort halten und neue Familien mit Kindern gewinnen zu können. Dafür müssen attraktive Kinderbetreuungs-, Bildungs- und Freizeiteinrichtungen auf kommunaler Ebene oder im Verbund mit anderen Gemeinden in der Kleinregion bereitgestellt werden.

Im Gestaltungsraum „,regionale Produkte“ gilt es zukünftig auf regionale Dachmarken zu setzen, die allerdings nur auf einer soliden einzelbetrieblichen Basis ihr volles Synergiepotenzial ausschöpfen können. So können Ressourcen in Produktion und Vermarktung gebündelt werden. Österreichische Gemeinden nutzen diese Möglichkeit bereits erfolgreich. Als Beispiele seien die Südsteirische Weinstraße oder die Vorarlberger Käsestraße genannt. Trotz des vergleichsweise kleinen Marktes gibt es auch in Slowenien schon einige Beispiele hochwertiger, überregional bekannter Lebensmittel und Handwerksprodukte, die in ländlichen Gemeinden hergestellt werden. Hier zeigen sich positive Synergieeffekte mit dem wachsenden Tourismusmarkt, der 
die Regionen international bekannter macht und damit neue Absatzmärkte für die ansässigen Betriebe öffnet.

Zur Unterstützung dieser Trends besteht Handlungsbedarf auch bei politischen Institutionen auf nationaler Ebene. Es geht darum, zum Beispiel durch Medienkampagnen das Konsumentenbewusstsein für heimische Produkte zu schärfen und so die lokale Nachfrage zu stimulieren. Hier kann ein Know-how-Transfer regionalpolitischer Maßnahmen aus Österreich in die Nachbarländer als sinnvoll erachtet werden. Man denke beispielsweise an die Medienkampagnen der Agrar.Markt.Austria oder des Lebensministeriums. Die Betriebe im ländlichen Raum der ostmitteleuropäischen Länder sollten dabei allerdings von den Erfahrungen ihrer Kollegen in den alten EUMitgliedsländern lernen: Die marktbeherrschenden Handelsketten werden sich vermutlich auch in den Transformationsländern an die Spitze eines Regionalitätstrends setzen wollen. Deshalb müssen frühzeitig wirtschaftspolitische Maßnahmen ergriffen werden, um durch regionale Kooperationsinitiativen landwirtschaftliche und gewerbliche Klein- und Mittelunternehmen im Wettbewerb zu stärken (RössL et al. 2006). Hinderlich erscheinen dabei sicher die Erfahrungen der Akteure mit genossenschaftlichen Zwangsorganisationen in kommunistischer Zeit.

Das Marketing für regionale Produkte ist derzeit sicher noch zu wenig professionell. Ebenso sind die Produktionskriterien und Qualitätssicherungsinstrumente noch nicht weit genug entwickelt. Regionale Produkte sollten stärker mit bekannten Regionsnamen und positiv belegten regionalen Gegebenheiten (Natur, Geschichte etc.) verbunden werden, um beim Kunden positive Assoziationen hervorzurufen. Zu dieser Marketingstrategie gehört auch die Revitalisierung traditioneller regionaler Rohstoffe und Produkte, die oft bereits überregional mit positiven Imageattributen versehen sind.

Der Gestaltungsraum „Beratung von Unternehmen“ stellt Gemeinden zukünftig vor große Herausforderungen. Ihre Kernkompetenz besteht bisher in den Themen Flächenwidmung, kommunale Steuerangelegenheiten oder Baugenehmigungen. Darüber hinaus geht es in Zukunft vermehrt um die Weitervermittlung an regionale Behörden, Kammern oder Beratungsinstitutionen für Spezialfragen. Wo solche Institutionen in der Ansiedlungsberatung tätig sind, sind sie und nicht mehr die Gemeinden erste Anlaufstelle für Gründer und ansiedlungswillige Unternehmer. Sie werden damit bei der (Jung-)Unternehmer- und Förderberatung zu wichtigen Kooperationspartnern für die Gemeinden. Die Beratung sollte allerdings so unkompliziert und kostengünstig wie möglich organisiert sein.

Dafür ist eine bessere Abstimmung zwischen den für Unternehmensfragen betroffenen Instanzen der Verwaltung bzw. zwischen staatlichen und nicht-staatlichen Beratungseinrichtungen notwendig. Vorab ist die Kompetenzverteilung zu klären. Es ist sicherzustellen, dass es eine Internetseite oder Informationsstelle gibt, die einerseits für die Gemeinden selbst und andererseits direkt für die Unternehmen tagesaktuell über die relevante Gesetzeslage informiert.

Die Gemeindeführungen müssen sich zukünftig jedenfalls als kompetente Partner für die lokalen Unternehmer platzieren, werden dabei aber sicher nie für das gesamte Spektrum an Fragen und Anliegen aus der Unternehmerschaft zuständig und kompetent sein können. Den Unternehmern sollte eine Kleinregion gegenüberstehen, deren 
Gemeinden sich in Fragen der Standortpolitik regelmäßig abstimmen und Beratungsressourcen bündeln. Sinnvoll wäre beispielsweise eine gemeinsame Ansprechperson für Unternehmensansiedlung in der Kleinregion.

Im Gestaltungsraum ,pluralistische Gemeindeentwicklung“ gilt es die Bevölkerung in den politischen Prozess zu integrieren. Um die Zivilgesellschaft zu stärken, sollten unter anderem regelmäßige Bürgerversammlungen zu bestimmten Themen abgehalten werden. Die gemeindepolitischen Entscheidungen müssen transparenter werden, und die Bevölkerung muss man aktiver in Entwicklungsprozesse einbinden. Die Akzeptanz der Ergebnisse würde jedenfalls erhöht und politisches Interesse - gerade auch bei der jungen Bevölkerung - geweckt.

Pluralistische und demokratische Einstellungen von Akteuren in den Gemeinden stehen wiederum in engem Zusammenhang mit den involvierten Generationen. Vor allem sollte der Einstieg einer jungen Kommunalpolitikergeneration verstärkt gefördert werden, da hier kreatives Potenzial zur Lösung aktueller Probleme schlummert. Dazu muss es für die Jungen aber auch wieder attraktiv werden, sich in der Gemeindepolitik zu engagieren. Das kann beispielsweise durch deren frühzeitige Einbindung in Entscheidungsprozesse gefördert werden.

Wenn die Entscheidungsträger eine demokratische und offene Grundeinstellung haben, äußert sich das oft in einer Reihe von Maßnahmen zur Verbesserung der Transparenz in der Gemeindepolitik: regelmäßig einberufene Bürgerforen, „offene Tür“ im Gemeindeamt, Gemeindepublikationen mit unterschiedlichen Sichtweisen auf die Gemeindeentwicklung, transparente Informationspolitik über Absichten und Beschlüsse der Gemeinde, individuelle Bürgergespräche, Diskussionsforen im Internet.

Wie auch in den „,alten“ EU-Ländern sucht man als Konsequenz aus Staats- und Marktversagen nach neuen horizontalen Steuerungsstrukturen in der Gemeinde- und Regionalentwicklung außerhalb der etablierten Institutionen. Damit rückt der Gestaltungsraum ,interkommunale Kooperation“ zunehmend in den Fokus der Gemeindeentwicklung. Diese Initiativen münden in der Zusammenarbeit von staatlichen Akteuren mit Betrieben und Organisationen der Zivilgesellschaft. Ob die aktuelle politische Debatte über good governance als Schlagwort hinausgeht und tatsächlich zur Etablierung neuer demokratischer und pluralistischer Steuerungsstrukturen führt, darf - nicht nur für den Untersuchungsraum der Studie, sondern für die gesamte EU - durchaus mit Skepsis gesehen werden. Wenn, dann scheint allerdings die Gemeinde oder die Kleinregion aufgrund der geringeren Komplexität noch am ehesten für derartige Steuerungsmodelle geeignet zu sein. Im Gegensatz zu Österreich gibt es in den Transformationsländern jedenfalls keine Tradition eines gesellschaftlich-liberalen Korporatismus, wie er beispielsweise durch die österreichische Sozialpartnerschaft repräsentiert wird. Demgegenüber steht jedoch eine schlagkräftigere Tradition von zivilgesellschaftlichen Bewegungen.

Die Gemeinden könnten beim Aufbau zwischenbetrieblicher Kooperationen in Abstimmung mit den auf diesem Feld bereits aktiven Regionalberatungen durchaus eine Schlüsselrolle einnehmen. Kooperationen zwischen Unternehmern in der Region sollten unterstützt werden. Entsprechende Maßnahmen wären die Organisation von 
Informationsveranstaltungen, die Weitervermittlung an kompetente Kooperationsbegleiter oder auch die Förderung lokaler Verkaufsstellen wie sie im Untersuchungsraum bereits vereinzelt anzutreffen sind. Dabei wirkt sich immer noch hemmend aus, dass Begriffe wie „Kooperation“ oder „Genossenschaft“ aus kommunistischer Zeit negativ besetzt sind und die Rolle von Umfeldakteuren (z.B. Regionalpolitikern, Interessenvertretungen) mitunter skeptisch gesehen wird.

Schließlich sollte auch in der Bildungspolitik der Hebel für regionale Innovationen angesetzt werden. Hier gilt es die jüngere Bevölkerung für das Unternehmertum $\mathrm{zu}$ begeistern. Bildung hatte in den ostmitteleuropäischen Ländern schon während des Kommunismus einen hohen Stellenwert. Nun gilt es die Schulen als Träger des lokalen Know-hows auch für Fragen des Unternehmertums und der Innovation zu nutzen. Der Austausch zwischen ansässigen Unternehmern und lokalen Bildungseinrichtungen sollte forciert werden. In die Schulen können die Unternehmer die lokalen Wirtschaftstraditionen einbringen. Umgekehrt können Bildungseinrichtungen die praktische Erfahrung der Unternehmer mit aktuellem Wissen anreichern. Dabei sollte die Vermittlung eines modernen Unternehmertums angestrebt werden, das der Pflege der lokalen Wirtschaftstradition verpflichtet ist, die lokalen Gegebenheiten als unternehmerische Gelegenheiten zu nützen versteht und diese Besonderheiten als Wettbewerbsvorteile kommunizieren kann. In diesem Zusammenhang bieten sich auch Businessplanwettbewerbe zum Thema „nachhaltige Regionalentwicklung“ an. Dennoch werden Strategien in der betriebswirtschaftlichen Aus- und Weiterbildung nur dann erfolgreich sein, wenn in diesem Bereich eine Zusammenarbeit zwischen den Gemeinden auf regionaler Ebene gelingt.

Wenn es um interkommunale Kooperation geht, erscheint im Untersuchungsraum auch die Zusammenlegung der Verwaltungseinrichtungen notwendig. Die fachliche Ausbildung von Gemeinderäten und -bediensteten reicht oft nicht aus, um fundierte Entscheidungen für eine nachhaltige Gemeindeentwicklung treffen zu können (z.B. Betriebsansiedlungen, Sozial- und Ausbildungsmaßnahmen). Die regionalen Potenziale für den Aufbau notwendiger Fachkompetenzen (z.B. Betriebswirtschaft, Recht) sollten daher gebündelt werden. Größere Verwaltungseinheiten verfügen über ausreichende Ressourcen, um die Qualität der Entscheidungen und Beratung gewährleisten zu können. Allerdings ist auf die Gefahr bürokratischer Trägheit hinzuweisen.

Eine verstärkte Verwaltungszusammenlegung ist überdies auch aus Effizienzgründen sinnvoll. Die Gemeinden im Untersuchung sraum leiden unter notorischen Budgetproblemen. Durch interkommunale Gemeindeämter und Schulzentren könnten Budgetmittel eingespart werden. Wenn allerdings die Qualität der angebotenen regionalen Leistungen nicht verbessert wird, besteht die Gefahr verminderten Lebensstandards der Bewohner in den einzelnen Gemeinden, weil sie die entsprechende örtliche Infrastruktur verlieren. Zudem droht bei Behördenzusammenlegungen der Verlust lokaler Arbeitsplätze.

Wenn Infrastruktureinrichtungen zusammengelegt werden, versuchen Bürgermeister zumeist, sie in der eigenen Gemeinde anzusiedeln. Zusammenlegungen sind daher oft nur durchsetzbar, wenn die Regionalentwicklung noch zentralistisch organisiert ist. Dezentralisierung und ein höheres $\mathrm{Ma}$ an Gemeindeautonomie fördern also zwar Bürokratieabbau und Flexibilität, unterstützen aber auch den Wettbewerb zwischen den Gemeinden um Unternehmensansiedlungen, wofür auch viele Fördermittel eingesetzt 
werden. Wichtige Fragen der Lebensqualität der örtlichen Bevölkerung (z.B. soziale Sicherheit, günstige Wohngründe) bleiben damit häufig auf der Strecke.

Um die interkommunale Zusammenarbeit im Untersuchungsraum nachhaltig zu verankern, wäre es wichtig, die Interaktion zwischen Vertretern der Gemeinden einer Region zu institutionalisieren. Als positives Beispiel kann ein regelmäßiger Bürgermeisterrat in den slowenischen Kleinregionen genannt werden. Es dürfen auch nicht Fördermittel der wichtigste Anreiz für Kooperationen sein. Nur wenn die Vorteile der regionalen Zusammenarbeit allen Beteiligten bewusst sind, kann sie Bestand haben.

Wie aber können Impulse von außen so gesetzt werden, dass sie von der Bevölkerung und der Unternehmerschaft nicht als Zwang empfunden werden? Ein negatives Beispiel ist in dieser Hinsicht die von staatlicher Seite angeordnete Neustrukturierung der Regionen in Ungarn und Slowenien. Sie erinnert an Vorgangsweisen zur Zeit des Kommunismus und fördert nicht die regionalen Kooperationskulturen, da sie von der örtlichen Bevölkerung nicht mitgetragen wird.

Regionale Kooperationskultur zu fördern wird am ehesten durch ein regional verankertes Projektmanagement gelingen, das von den Betroffenen als unabhängig empfunden wird. Es sollte allerdings finanziell und fachlich über ausreichende Ressourcen verfügen. Die so erarbeiteten Projekte wären in die Gemeindeentwicklungspläne einzubetten und interkommunal verbindlich abzustimmen.

\subsection{Diskussion der Ergebnisse}

Ein funktionierendes Wirtschaftssystem ist mehr als freier Wettbewerb!

Um die strukturellen Rahmenbedingungen des Handelns auf kommunaler Ebene $\mathrm{zu}$ verstehen, muss der Fokus der Betrachtung auf Entwicklungstendenzen auf der Makroebene erweitert werden. Gerade auf diese haben die Gemeinden im gesamten Untersuchungsraum jedoch nur bedingt Einfluss.

Der Staat verlässt sich bei der Entwicklung einer leistungsfähigen Wirtschaftsstruktur - auch weil er heute keine Alternative mehr sieht - stärker auf private Investoren. Angesichts kleiner werdender Haushaltsspielräume bei gleich bleibenden oder sogar größer werdenden Aufgaben sind die Bürgermeister wie in ganz Europa zu Privatisierungen öffentlicher Dienstleistungen oder zum Eingehen von Public Private Partnerships (PPP) gezwungen (HoltKAMP 2007). Für größere private (ausländische) Investoren ist der infrastrukturell benachteiligte ländliche Raum allerdings ohne massive Förderungen kaum attraktiv. Durch die derzeit gelebte Dumpingstrategie im interkommunalen Standortwettbewerb können ressourcenschwache Gemeinden aber kaum Einfluss darauf nehmen, wo sich ein größeres Unternehmen ansiedelt. Zugleich ist heute jeder Standort nach dem Auslaufen von öffentlichen Förderungen massiv bedroht. Dies zeigt drastisch die Schließung des profitablen Nokia-Werks im deutschen Bochum und die Verlagerung der Produktion in das rumänische Jucu bei Klausenburg [Cluj-Napoca], wodurch ca. 2.000 deutsche Mitarbeiter ihren Arbeitsplatz verloren, obwohl in den Standort Bochum rund 88 Mio. Euro an öffentlicher Förderung geflossen waren. Dies zeigt deutlich die Machtlosigkeit von Standortpolitik mittels Förderungen gegenüber kurzfristigen Unternehmensstrategien, welche die soziale Sicherung am Standort nicht mehr berücksichtigen (MALVACHE 2008). 
Die Abhängigkeit von privaten Investoren bei der Entwicklung von Gemeinden wirft auch das demokratiepolitische Problem auf, dass die Bevölkerung kaum noch auf Entscheidungen und Programme Einfluss nehmen kann. Weil sich die öffentliche Hand aus zahlreichen kommunalen Aufgaben zurückzieht, nehmen private Investoren oft einseitigen Einfluss, bedrohen damit das soziale Gefüge und nähren die Frustration der Bevölkerung in Bezug auf Gemeindeentwicklung.

Aus den Fehlern der liberalen Marktwirtschaften in den „,alten“ EU-Ländern wurde in den untersuchten Transformationsländern offensichtlich wenig gelernt. Als Gegenreaktion auf das individualismusfeindliche und die Eigeninitiative unterdrückende kommunistische System setzt sich ein bedingungsloser Glaube an die Selbstregulierungskraft des Marktes durch, der mitunter in einer radikalen Umsetzung des Laissez-faire-Gedankens mündet. Neo-liberale Tendenzen innerhalb der Gesellschaft erscheinen somit in den ostmitteleuropäischen Ländern stärker wirksam zu sein als in Österreich, da der Wettbewerbsmaxime größere Lebensbereiche unterworfen werden (Segert 2009; Steger 2006).

Der Transfer von lokalen Entwicklungsstrategien über nationalstaatliche Grenzen hinweg, und damit auch die Gefahr Fehler zu wiederholen, wird durch die Vorgaben im Rahmen der gemeinsamen europäischen Regionalpolitik verstärkt. Doch motivieren gerade europäische Fördertöpfe stärker als alle nationalen Förderinstrumente zur Aktivität in der Regionalentwicklung. Sie sind die treibende Kraft für interkommunale Kooperation im Untersuchungsraum. Aus der Studie wird deutlich, dass ohne die Maßnahmen und Anreize der europäischen Regional- und Strukturpolitik Zusammenarbeit zwischen den ländlichen Gemeinden nicht in diesem Ausmaß stattfinden würde.

\section{Ähnlichkeiten der Herausforderungen in der Gemeindeentwicklung führen zu einer mitteleuropäischen Perspektive}

Die Ergebnisse der Studie zeigen, dass Österreich und die ostmitteleuropäischen Länder hinsichtlich ihrer Rahmenbedingungen für wirtschaftspolitische Maßnahmen auf kommunaler Ebene größere Ähnlichkeiten aufweisen als man gemeinhin annehmen würde. So ist es gerade der Prozess der Globalisierung, welcher die ländlich geprägten Gemeinden im mitteleuropäischen Raum mit ganz ähnlichen Herausforderungen bei der Schaffung von Rahmenbedingungen für Klein- und Mittelunternehmen konfrontiert, wenn die Problemlagen auch unterschiedlich scharf hervortreten. Die Folgen neo-liberaler Wirtschaftspolitik sind hier wie dort spürbar, indem sich der Staat aus seiner Regulierungsfunktion in Wirtschaft und Gesellschaft zurückzieht (SEGERT 2009).

Es spricht Vieles dafür, Mitteleuropa verstärkt als einen gemeinsamen Entwicklungsraum zu betrachten, in dem sich die Kleinregionen aufgrund ähnlicher Problemlagen vernetzen und auch grenzüberschreitende Entwicklungsstrategien ausarbeiten. Bei der Raumentwicklung wirken dabei verschiedene Ebenen mit, auf welchen jeweils spezifische Maßnahmenbündel zur Etablierung einer mitteleuropäischen Regionalentwicklung umgesetzt werden müssen. Ein möglicher Ansatzpunkt dazu könnte das Interreg-Programm sein, eine Gemeinschaftsinitiative des Europäischen Fonds für Regionale Entwicklung (EFRE) zur Förderung der Zusammenarbeit zwischen den Regionen der EU. 
International agierende Unternehmen haben Gemeinsamkeiten der Regionen im mitteleuropäischen Raum im Rahmen ihrer Marktbearbeitungsstrategien schon früh erkannt und, in ihrem Sinne, erfolgreich berücksichtigt. Es ist an der Zeit, dass diese Sichtweise auch von der Politik der betroffenen Nationalstaaten internalisiert wird und gemeinsame Strategien für die wirtschaftliche Entwicklung auf kommunaler Ebene folgen. Um das zu ermöglichen, muss jedoch der nationalstaatliche Egozentrismus sowie das Kirchturmdenken regionaler und kommunaler Entscheidungsträger überwunden werden. Jede Gemeindeführung sollte das eigene Handeln in einem größeren Ganzen verstehen. Identitätsstiftend sollten dabei nicht mehr die Abgrenzung zu anderen Räumen, sondern die Gemeinsamkeiten sein. Dazu sind von europäischer und nationaler Ebene weitere Anreize zu schaffen, um eine grenzüberschreitende Vernetzung der ländlichen Gemeinden in Mitteleuropa zu fördern.

Wechselseitiger Wissenstransfer heißt kritische Auseinandersetzung mit den eigenen Gegebenheiten als Grundlage für die Übernahme erfolgreicher Muster

Ausgehend von den eben dargelegten Argumenten sollte es zwischen den Gemeinden in Mitteleuropa zu einem wechselseitigen Know-how-Transfer kommen, der allerdings nicht die bloße Übertragung von Entwicklungskonzepten von einer Kleinregion auf die andere zum Ziel haben darf. Kleinregionen sollten vielmehr individuell an ihre Gegebenheiten und Bedürfnisse angepasste Entwicklungsstrategien definieren und sich nicht - z.B. aufgrund von Förderrichtlinien - an Kennzahlen von Vergleichsregionen anpassen und damit deren Strukturen unreflektiert übernehmen.

Gerade in den ostmitteleuropäischen Ländern sind die negativen Folgen standardisierter, „am Reißbrett“ entwickelter regionalpolitischer Konzepte zu beobachten. Diese Konzepte wurden über die Räume „gestülpt“, ohne die ansässige Bevölkerung einzubeziehen. Statt einer Vervielfältigung von Maßnahmen, die in anderen Gemeinden erfolgreich waren, bedarf es einer grenzüberschreitenden Wissensvermittlung, die den Gemeinden in erster Linie das verfügbare Instrumentarium kommunaler Wirtschaftspolitik nahe bringt. Der Know-how-Transfer sollte den Gemeinden nicht den Entwicklungspfad vorgeben, sondern Wege aufzeigen, wie jede Gemeinde ihren individuellen Entwicklungspfad finden und konsequent verfolgen kann.

Dabei können auch die österreichischen Gemeindevertreter von ihren Kollegen in den ostmitteleuropäischen Ländern lernen, z.B. wie Gemeindepolitik unter schwierigen Bedingungen betrieben werden kann. In der Ära des Kommunismus und später in den Jahren der Transformation waren die Gemeinden dieser Länder in besonderem Maße mit dem Problem knapper Ressourcen konfrontiert. Auch die Erfahrungen aus dem Transformationsprozess können österreichischen Gemeinden, die mit strukturellen Umbrüchen ihrer Wirtschaftsstruktur konfrontiert sind, wertvolle Hinweise bieten.

Bei der Bewertung der Übertragbarkeit erfolgreicher wirtschaftspolitischer Maßnahmen in den Gemeinden darf nicht auf die Problematik der kontextuellen Einbettung von Strategien vergessen werden. Sowohl die Maßnahmen der Gemeinden als auch die der Unternehmen entfalten erst im spezifischen Kontext ihre Wirkung. Wie die Faktoren in den Bereichen Verwaltung, Unternehmen, Bevölkerung jeweils ineinander greifen und welche Einflüsse auf die Kleinregion von außen einwirken, entscheidet darüber, ob die Kleinregion eine erfolgreiche Entwicklung nimmt. 
Daher müssen positive Erfahrungen in der Gemeindeentwicklung zuerst aus ihrem ursprünglichen Entstehungskontext herausgelöst werden, um sie übertragbar zu machen. Es müssen die hinter den Entwicklungen stehenden Ursachengefüge verstanden und die besonderen örtlichen Voraussetzungen einer erfolgreichen Entwicklung erkannt werden. Dabei ist zu berücksichtigen, dass es sich bei Gemeinden um komplexe soziale Systeme handelt (LUHMANn 1984; vON FOERSTER 1981), wodurch Interventionen nur vor dem Hintergrund eines systemischen Verständnisses (KöNIGSWIESER \& EXNER 2001) gesetzt werden können. Punktuelle Intervention wird somit unmöglich, und der Eingriff in das System kann nur durch Impulse (Irritationen), die der Logik der internen Kräfte des Systems entsprechen, erfolgen (BAECKER 2003).

Jede Maßnahme darf daher nur als abstrakter Strategietyp verstanden werden, der von den Akteuren in der Kleinregion erst konkretisiert werden muss. So verstanden, erfordert der grenzüberschreitende Wissenstransfer zwischen Gemeinden eine kritische Auseinandersetzung mit erfolgreichen Mustern. Die Einbettung einer Maßnahme in den spezifischen Kontext liegt sowohl in der Verantwortung der Entscheidungsträger als auch der Betroffenen.

\section{Literaturverzeichnis}

B AECKER D. (2003), Organisation und Management. Frankfurt am Main, Suhrkamp.

Becker D. (2009), Die Sache mit der Führung. Wiener Vorlesungen. Wien, Picus.

BENDER K.W. (1997), Entrepreneurship and Local Development in Countries in Transition. In: Entrepreneurship and SMEs in Transition Economies. The Visegrad Conference, S. 167-174. Paris, OECD

Bouckenooghe D., De Clerce D., Willem A., Buelens M. (2007), An Assessment of Validity in Entrepreneurship Research. In: The Journal of Entrepreneurship, 16, 2, S. 147-171.

Chandler C., Lyon D. (2001), Issues of Research Design and Construct Measurement in Entrepreneurship Research: The Past Decade. In: Entrepreneurship: Theory \& Practice, 25,4 , S. 101-113.

Diekmann A. (2000), Empirische Sozialforschung. Grundlagen, Methoden, Anwendungen. Reinbek bei Hamburg, Rowohlt.

Du Plessis V., Beshiri R., Bollmann R.D., Clemenson H. (2002), Definitions of „Rural“, Agriculture and Rural Working Paper Series. In: Working Paper No. 61, Ottawa, Statistics Canada.

Egner H. (2006), Autopoiesis, Form und Beobachtung. Moderne Systemtheorie und ihr möglicher Beitrag für eine Integration von Human- und Physiogeographie. In: Mitt. d. Österr. Geogr. Ges., 148, 1, S. 92-108.

Essmann H. (1980), Zur Entwicklung des Ländlichen Raumes in Österreich. Ergebnisse einer Strukturuntersuchung und Folgerungen für die Raumordnungspolitik. Salzburg, Salzburger Inst. f. Raumforschung.

Europäische Kommission (2003), Empfehlung der Kommission vom 6. Mai 2003 betreffend die Definition der Kleinstunternehmen sowie der kleinen und mittleren Unternehmen, AB1. L 124, S. 36-41. 
Fassmann H., Meusburger P. (1997), Arbeitsmarktgeographie. Erwerbstätigkeit und Arbeitslosigkeit im räumlichen Kontext. Stuttgart, B.G. Teubner.

FinK M. (2005), Erfolgsfaktor „Selbstverpflichtung“ bei vertrauensbasierten Kooperationen Mit einem empirischen Befund. Frankfurt am Main, Lang.

Gopinath C., Hoffman R.C. (1995), A comment on the relevance of strategy research. In: Shrivastava P., Huff A.S., Dutton J.E. (Hrsg.), Advances in Strategic Management: Challenges from within the Mainstream, S. 93-110. Greenwich, CT, JAI Press.

GRICHNIK D. (2006), Die Opportunity Map der internationalen Entrepreneurshipforschung. In: Zeitschrift f. Betriebswirtschaft, 76, 12, S. 100-125.

Harms R., Kraus S., Schwarz E. (2009), The suitability of the configuration approach in entrepreneurship research. In: Entrepreneurship and Regional Development, 21, 1, S. 25-47.

Henkel G. (1993), Der Ländliche Raum. Gegenwart und Wandlungsprozesse in Deutschland seit dem 19. Jahrhundert. Stuttgart, B.G. Teubner.

Hitt M.A., Ahlstrom D., Dacin M.T., Levitas E., Svobodina L. (2004), The Institutional Effects on Strategic Alliance Partner Selection in Transition Economies: China vs. Russia. In: Organization Science, 15, 2, S. 173-186.

Holtkamp L. (2007), Local Governance. In: Benz A. (Hrsg.), Handbuch Governance: theoretische Grundlagen und empirische Anwendungsfelder. Wiesbaden, Verl. f. Sozialwiss.

Königswieser R., ExNer A. (2001), Systemische Intervention. Architekturen und Designs für Berater und Veränderungsmanager. Stuttgart, Klett Cotta.

LANGOSCH R. (1994), Theoretische Grundlagen für die Analyse von innovationsbedingten Unterschieden des wirtschaftlichen Wachstums in ländlichen Räumen. Frankfurt am Main et al., Peter Lang.

Luhmann N. (1984), Soziale Systeme: Grundriss einer allgemeinen Theorie. Frankfurt am Main, Suhrkamp.

Luhmann N. (1995), Die operative Geschlossenheit psychischer und sozialer Systeme. In: LuHMANN N. (Hrsg.), Die Soziologie und der Mensch, S. 25-36. Opladen, Westdt. Verlag.

Malvache J. (2008), Die Ansiedlung von Nokia in Cluj (Rumänien): Globalisierung im europäischen Kontext, Perspektiven des Demokratischen Sozialismus. In: Zeitschrift f. Gesellschaftsanalyse u. Reformpolitik, 1, 25, S. 115-137.

MaYRING P. (2002), Einführung in die qualitative Sozialforschung; Eine Anleitung zu qualitativem Denken. Basel, Weinheim.

Mugler J. (2005), Grundlagen der BWL der Klein- und Mittelbetriebe. Wien, Facultas.

OECD (Hrsg.) (1993), Welche Zukunft haben unsere ländlichen Räume? Eine Politik der ländlichen Entwicklung. Paris.

OECD (Hrsg.) (1995), Creating Employment for Rural Development. New Policy Approaches. Paris.

Rössl D., Berger G., Fink M., LANG R. (2006), The Evolution of Co-operation and Co-operatives between Agricultural and Commercial Enterprises. Intern. Conf. d. UNWE and CCU, Sofia, Bulgarien

Rudolph A. (1997), Die Bedeutung von Handwerk und Kleinunternehmen für die Regionalpolitik. Eine theoretische und empirische Betrachtung (= Göttinger Handwerkstudien, 51). Duderstadt.

Rust H. (1981), Methoden und Probleme der Inhaltsanalyse. Tübingen, Narr.

Schmalhaus S., Stember J. (1993), Schaffung gleichwertiger Lebensverhältnisse im ländlichen Raum. Möglichkeiten und Grenzen der Raumordnung. In: Schmalhaus S., Stember J. (Hrsg.), Entwicklungsprobleme im ländlichen Raum, S. 3-24. Münster - Hamburg, Lit.

SсHÖN H. (1997), Regionalpolitische Konzepte und Strukturwandel ländlicher Räume. Eine Analyse am Beispiel des oberen Altmühltals. Berlin, Duncker \& Humblot. 
SchütZe F. (1987), Das narrative Interview in Interaktionsfeldstudien. Hagen.

Segert D. (2009), Die Politik der post-kommunistischen Linken in Mittel- und Osteuropa: Der Einfluss auf die Konsolidierung oder Gefährdung der Demokratie. In: B ACKES U., Jaskulowski T., Polese A. (Hrsg.), Totalitarismus und Transformation: Defizite der Demokratiekonsolidierung in Mittel- und Osteuropa, S. 119-134. Göttingen, Vandenhoeck \& Ruprecht.

Steger T. (2006), Auf dem Weg zum Neo-Liberalismus? Ein kritischer Blick auf die Entwicklung der industriellen Beziehungen in Mittel- und Osteuropa. In: JurczeK P., NiedobiteK M. (Hrsg.), Europäische Forschungsperspektiven - Elemente einer Europawissenschaft, S. 153-172. Berlin, Duncker \& Humblot.

Tsang E., Kwan K.-M. (1999), Replication and Theory Development in Organization Science: A Critical Realist Perspective. In: Academy of Management Review, 24, 4, S. 759-780.

V AN DE VEN A.H. (2002), Strategic directions for the Academy of Management: this academy is for you. In: Academy of Management Review, 27, 2, S. 171-184.

Veliyath R., SRinivasan T.C. (1995), Gestalt approaches to assessing strategic coalignment: A conceptual integration'. In: British Journal of Management, 6, 3, S. 205-219.

Von Foerster A. (1981), Observing Systems. Seaside, Intersystems Publ.

Wiklund J., ShePherd D. (2005), Entrepreneurial Orientation and Small Business Performance: A Configurational Approach. In: Journal of Business Venturing, 20, 1, S. 71-91. 